'Departamento de Psicología.

Laboratorio de Estrés y Salud.

Universidad de La Frontera. Temuco, Chile.

aPsicólogo, Doctor en Psicología.

'Psicólogo, Ph.D. Doctor en Psicología de la Salud.

Trabajo financiado por CONICYT Programa de Formación de

Capital Humano Avanzado/ Doctorado Nacional 21150016, y Proyecto FONDECYT de Iniciación No11140454.

Los autores declaran no tener conflictos de interés.

Recibido el 9 de octubre de 2018, aceptado el 29 de marzo de 2019.

Correspondencia: Daniela Gómez-Pérez. Montevideo 0830, Temuco, Chile. daniela.gomez@ufrontera.cl

\section{Estigma de obesidad, cortisol e ingesta alimentaria: un estudio experimental con mujeres}

\author{
DANIELA GÓMEZ-PÉREZ ${ }^{1, a}$, MANUEL S. ORTIZ ${ }^{1, b}$
}

\section{Association between weight-based stigmatization psychological stress and calorie intake}

\begin{abstract}
Background: Weight-based stigmatization is frequent among overweight and obese people. Aim: To determine the association between weight-based stigmatization, psychological stress, cortisol, negative emotions, and eating behavior in a sample of middle-aged women. Material and Methods: Eighty-two women aged $45 \pm 8$ years, $55 \%$ with overweight or obesity, were randomly allocated to watch a video called "Stigma: the human cost of obesity" or a control video about planet earth. The effect of watching either video on calorie consumption, psychological stress and cortisol reactivity was assessed. Cortisol was measured on four salivary samples. Psychological stress and negative emotions were self-reported. Results: Among women who watched the stigmatizing video, there was a direct association between psychological stress and calorie intake, but negative emotions did not mediate this association. Moreover, psychological stress moderated the association between watching the stigmatizing video and the cortisol output ( $\beta=0.32 ; p=0.005)$. Conclusions: Women with high psychological stress have a greater intake of calories. After watching the stigmatizing video, a greater psychological stress is associated with greater cortisol output.
\end{abstract}

(Rev Med Chile 2019; 147: 314-321)

Key words: Discrimination (Psychology); Feeding Behavior; Glucocorticoids; Obesity; Psychological Stress.

\section{L}

a obesidad es una epidemia a nivel mundial, con alta prevalencia tanto en países desarrollados como en vías de desarrollo ${ }^{1}$. Tiene graves consecuencias para la salud de los individuos ${ }^{2}$ y genera altos costos médicos, sociales y económicos ${ }^{3}$. En Chile, el sobrepeso afecta al $39,8 \%$ de la población y la obesidad al $31,2 \%{ }^{4}$, por lo que el exceso de peso se ha convertido en un problema de salud con una tendencia generalizada al alza, especialmente en mujeres chilenas ${ }^{5}$.

La obesidad se produce por un desbalance entre ingesta calórica y consumo energético ${ }^{6}$, sin embargo, esta explicación puede ser insuficiente, ya que en la actualidad se evidencia una etiología multifactorial de la obesidad ${ }^{7,8}$, es decir, factores físicos, biológicos y psicológicos interactúan en su desarrollo ${ }^{9}$, por ejemplo, a nivel hormonal, la grelina y el cortisol se han asociado con el apetito $^{10}$ y a nivel conductual se ha descrito que un ambiente obesogénico podría asociarse con el aprendizaje y mantención de conductas tendientes a la ganancia de peso $^{11}$.

Respecto a las variables psicológicas, en la última década se ha comenzado a estudiar el rol que el estigma de obesidad tiene en la etiología del sobrepeso y la obesidad ${ }^{12-15}$, demostrándose que este podría relacionarse con el exceso de peso tanto en un nivel fisiológico como conductual ${ }^{16,17}$. 
El estigma de obesidad se define como la devaluación y denigración social de personas que tienen exceso de peso, lo cual las convierte en víctimas de prejuicios, estereotipos negativos $\mathrm{y}$ discriminación ${ }^{18}$. Aunque las experiencias de estigmatización ocurren hacia ambos sexos, las mujeres reportan una mayor frecuencia de estas experiencias ${ }^{19}$ siendo ellas mayormente afectadas que los hombres ${ }^{20,21}$, debido a que es más probable que internalicen estos mensajes negativos ${ }^{22}$.

La evidencia reporta que las personas que experimentan situaciones de estigmatización por peso, presentan mayor estrés psicológico ${ }^{16,23}$ y mayor ingesta alimentaria ${ }^{24}$. Para comprender el mecanismo por el cual opera el estigma de obesidad, conviene describir el Modelo Cíclico de la Obesidad y el Estigma basado en el Peso (MCOEP) ${ }^{18}$. Este modelo plantea que el estigma de obesidad es un estresor, el cual una vez inducido desencadena una serie de respuestas psicológicas, fisiológicas y conductuales que provocan un aumento de peso o dificultad para perder peso. Específicamente, el estigma operaría vía activación del eje hipotalámico-hipofisiario-adrenal (HHA), el cual es un complejo sistema de respuesta hormonal que se activa por estresores físicos y psicológicos ${ }^{25}$. De esta manera, la exposición a situaciones de estigmatización se asociaría con mayor estrés percibido $\mathrm{y}$, por lo tanto, mayores niveles de cortisol ${ }^{26}$. Más aún, si se considera que las experiencias de estigmatización reúnen las características centrales de un evento estresor, tales como incontrolabilidad, impredictibilidad y amenaza al self social $^{27}$, es habitual que las víctimas del estigma presenten una respuesta fisiológica al estrés caracterizada por la activación del eje y una mayor producción de cortisol $^{27,28}$, los cuales se asocian con ganancia de peso $^{29}$.

La ingesta alimentaria es una estrategia común en respuesta al estrés o a emociones negativas ${ }^{30,31}$, de hecho surge en la literatura científica el concepto de comfort food, el cual corresponde a una conducta definida por el incremento de la ingesta de alimentos altos en grasa, azúcar o calorías, en respuesta a emociones negativas o a eventos estresantes ${ }^{31}$, de modo tal que es posible hipotetizar que sujetos expuestos a estigmatización, a nivel emocional experimentan emociones negativas y a nivel conductual presentan una ingesta alimentaria inadecuada.

Dado que la obesidad es más prevalente en mujeres, y las consecuencias de la estigmatización son más severas en este grupo, el propósito de este estudio fue determinar la relación causal entre estigma de obesidad, reactividad fisiológica al estrés psicológico, respuesta emocional negativa y la conducta de ingesta alimentaria en una muestra de mujeres.

\section{Material y Método}

\section{Participantes}

Ochenta y dos mujeres funcionarias de la Universidad de La Frontera, Chile, y participantes de un estudio FONDECYT de Iniciación (11140454) aceptaron voluntariamente participar de este estudio. La edad promedio fue de 45 años ( $\mathrm{DS}=8,4$ ), el $45 \%$ de ellas tuvo un índice de masa corporal (IMC) correspondiente a la categoría normopeso, el $32 \%$ sobrepeso y $23 \%$ obesidad. Los criterios de exclusión fueron encontrarse embarazadas y autorreportar un consumo problemático de alcohol $(>$ siete tragos por semana). Aquellas mujeres que reportaron consumir tabaco, se les indicó suspender el consumo durante el día que participaran en el estudio.

Cuarenta y dos participantes fueron asignadas aleatoriamente al grupo de estigma y 40 al grupo control. No hubo diferencias estadísticamente significativas entre las condiciones según IMC, nivel de estrés y promedio de las medidas de cortisol salival en ninguno de los tres tiempos (Tabla 1).

\section{Instrumentos y materiales}

Positive Affect and Negative Affect Schedule $(P A N A S)^{32}$ : Corresponde a una escala con 20 palabras destinada a medir afecto positivo (10) y negativo (10). En este estudio, tras realizarse un Análisis Factorial Exploratorio de los ítems de afecto negativo, estos se agruparon en dos factores, uno con 7 emociones relacionadas a la tristeza $(\alpha=0,78)$ y los restantes 3 ítems se agruparon en un factor denominado Irritabilidad $(\alpha=0,74)$. El formato de respuesta es de tipo Likert entre 1 y 5 , en donde $1=$ muy levemente o nada y $5=$ extremadamente. La consistencia interna de la escala total resultó ser adecuada $(\alpha=0,77)$.

Estrés psicológico: Tal como se ha hecho en un estudio previo ${ }^{33}$, esta variable fue medida con un único ítem, el cual indagó cuán ansiosas se sintieron las participantes al momento del estudio. Las 
opciones de respuesta variaron en una escala de 5 puntos, en donde $0=$ Nada y $4=$ Extremadamente.

Estímulo experimental: La condición experimental fue manipulada con la presentación de un video de 19 minutos, titulado "Stigma: the human cost of obesity", elaborado por la cadena de televisión HBO. Este video tiene audio en inglés y subtítulos en español.

Estímulo neutro: La condición control, consistió en la presentación de un extracto de 19 minutos de un documental elaborado por la cadena de televisión BBC, titulado "Planet Earth II". El video tiene audio en inglés y subtítulos en español.

Medidas antropométricas: El IMC fue estimado con la fórmula clásica: Peso [kilogramos]/ estatura $\left[\right.$ metros $\left.^{2}\right]$. Las medidas fueron obtenidas a través de una varilla de medición Seca 220, fabricada de perfil de aluminio robusto con reglilla de plástico abatible, la cual está combinada con una báscula que permite estimar el peso en kilogramos. Este instrumento corresponde a un producto médico-sanitario conforme a la directiva 93/42/CE.

Cortisol salival: Fueron obtenidas cuatro medidas a través de Salivette ${ }^{\circledR}$ de marca Sarstedt. Estas muestras fueron recolectadas cada 20 minutos según el procedimiento previsto. Se calculó un promedio total de cortisol, descartando la primera medida. El cortisol salival se determinó mediante un enzimoinmunoensayo competitivo de fase sólida (ELISA) mediante el autoanalizador Quanta-Lyser 240 (Inova Diagnostics, San Diego CA, USA), empleando el kit Salivary Cortisol (DRG International, Inc., USA).

Ingesta alimentaria: Cada participante tuvo la oportunidad de consumir alimentos no saludables (maní salado y chocolates). El consumo total fue calculado a través de una pesa digital y luego transformado a kilocalorías.

\section{Procedimiento}

Este estudio tuvo la aprobación del Comité Ético Científico de la Universidad de La Frontera. Todas las participantes fueron citadas a las 11:00 horas, controlando con esto el efecto de la liberación diurna de cortisol. Previamente fueron instruidas de no consumir tabaco, alcohol, ni bebidas con cafeína. Se solicitó a cada participante desayunar y/o consumir alimentos hasta dos horas antes del experimento. Cada procedimiento fue ejecutado por ayudantes de investigación debidamente capacitadas, quienes obtuvieron consenti- miento informado y corroboraron el cumplimiento de las indicaciones antes descritas. Las muestras de cortisol se recolectaron posterior a este procedimiento, con un intervalo de 20 minutos entre ellas. El video en ambas condiciones fue expuesto entre la primera y segunda medida de cortisol. Entre la tercera y cuarta medida se facilitó la colación. Una vez finalizado el experimento, cada participante recibió información adicional sobre el experimento y se le retribuyó con 10.000 pesos.

\section{Plan de análisis}

Se realizaron pruebas $t$ de muestras independientes para determinar diferencias entre los grupos en las variables de IMC, estrés y medidas de cortisol. Se realizó análisis de senderos para probar efectos directos entre las variables de estrés, irritabilidad e ingesta alimentaria, utilizando como variable de agrupación la condición (experimental/control). Finalmente, se ejecutó análisis de curvas de crecimiento para estimar diferencias en las trayectorias del promedio total de cortisol de las participantes, según la condición, el IMC y el nivel de estrés. Dado que el cortisol no distribuyó en forma normal, se procedió a log transformar esta variable para los análisis, los cuales fueron ejecutados con STATA (versión 14.1).

\section{Resultados}

Tal como se observa en la Figura 1, el estrés psicológico se relacionó directamente con la ingesta alimentaria en el grupo de estigma $(\beta=0,56, p=0,001)$ y no en el grupo control $(\beta=0,08, p=0,63)$. En ambos grupos, se observa que el estrés psicológico se asoció con irritabilidad (estigma $\beta=0,42, p=0,001$ y control $\beta=0,52, p=0,001)$, sin embargo, esta variable no se relacionó con ingesta alimentaria en ningún grupo (estigma $\beta=0,064, p=0,63$; control $\beta=0,098, p=0,59)$. El test de efectos indirectos, demuestra que el factor de irritabilidad no medió la relación entre estrés psicológico y consumo de alimentos (estigma $\beta_{\text {indirecto }}=0,02, \mathrm{p}=0,42$; control $\left.\beta_{\text {indirecto }}=0,04, p=0,36\right)$. El efecto total del estrés psicológico en la ingesta, resultó ser estadísticamente significativo sólo en la condición de estigma (estigma $\beta_{\text {total }}=0,58, \mathrm{p}=0,001$; control $\left.\beta_{\text {total }}=0,12, \mathrm{p}=0,38\right)$.

La Tabla 2, describe los resultados del análi- 
Estigma de obesidad y cortisol - D. Gómez-Pérez et al

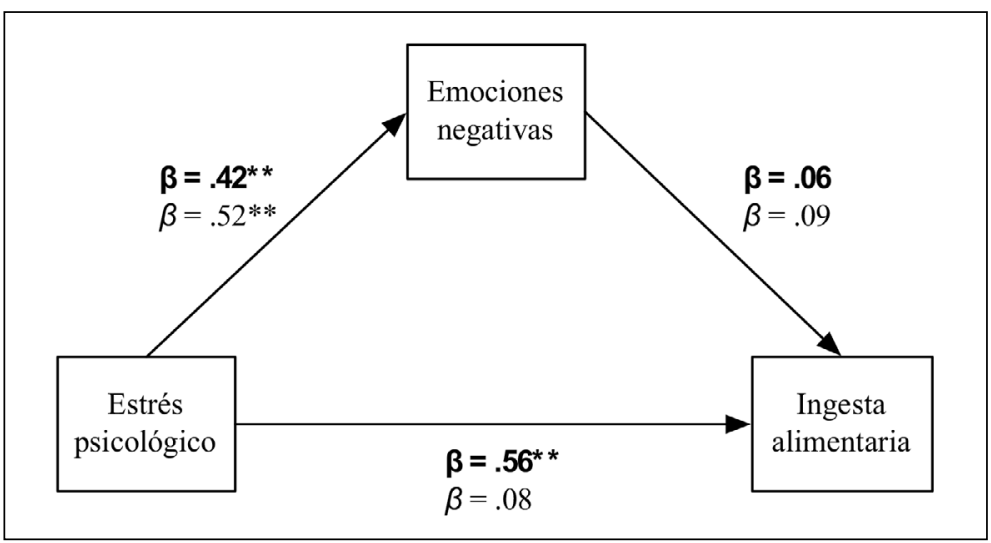

Figura 1. Modelo de relaciones propuesto entre estrés psicológico e ingesta alimentaria. Fuente: Elaboración propia. Negrita: resultados para grupo de estigma. Normal: resultados para grupo control. ${ }^{* *} p \leq 0,01$

Tabla 1. Datos descriptivos comparativos según condición

\begin{tabular}{|lrcccccc|} 
& \multicolumn{2}{c}{ Control } & \multicolumn{2}{c}{ Estigma } & & & \\
& M & DT & M & DT & gl & t & Sig. \\
IMC $\left(\mathrm{kg} / \mathrm{m}^{2}\right)$ & 26,6 & 0,7 & 26,1 & 0,6 & 80 & 0,52 & 0,6 \\
Estrés psicológico & 1,4 & 0,16 & 1,2 & 0,15 & 79 & 0,76 & 0,4 \\
Cortisol-1 $(\mathrm{ng} / \mathrm{mL})$ & 2,0 & 0,32 & 2,1 & 0,22 & 80 & $-0,37$ & 0,7 \\
Cortisol-2 $(\mathrm{ng} / \mathrm{mL})$ & 1,9 & 0,28 & 2,4 & 0,28 & 80 & $-1,17$ & 0,2 \\
Cortisol-3 $(\mathrm{ng} / \mathrm{mL})$ & 1,8 & 0,29 & 2,2 & 0,31 & 80 & $-0,92$ & 0,3 \\
\hline
\end{tabular}

Fuente: Elaboración propia.

Tabla 2. Trayectorias de cortisol y variables predictoras

\begin{tabular}{|lcccc|}
\hline Efectos fijos & $\boldsymbol{\beta}$ & $\mathbf{p}$ & $\mathbf{9 5 \%} \mathbf{C I}$ \\
\hline Estrés & $\mathbf{0 , 2 2 5}$ & $\mathbf{0 , 0 1 4}$ & $\mathbf{0 , 0 4 5}$ & $\mathbf{0 , 4 0 6}$ \\
\hline Irritabilidad & 0,209 & 0,368 & $-0,246$ & 0,664 \\
\hline Condición & 0,313 & 0,097 & $-0,057$ & 0,683 \\
\hline IMC & $\mathbf{- 0 , 4 0 6}$ & $\mathbf{0 , 0 2 8}$ & $\mathbf{- 0 , 7 6 8}$ & $\mathbf{- 0 , 0 4 3}$ \\
\hline Tiempo & $-0,076$ & 0,158 & $-0,183$ & 0,029 \\
\hline Estrés X Estigma & $\mathbf{0 , 3 1 9}$ & $\mathbf{0 , 0 0 5}$ & $\mathbf{0 , 0 9 7}$ & $\mathbf{0 , 5 4 1}$ \\
\hline Estrés X Control & 0,132 & 0,202 & $-0,070$ & 0,334 \\
\hline Constante & $-0,102$ & 0,752 & $-0,735$ & 0,530 \\
\hline Efectos aleatorios & & & 0,077 & 0,246 \\
$\sigma^{2}$ tiempo & 0,138 & - & 0,360 & 0,825 \\
$\sigma^{2}$ constante & 0,545 & - & $-0,082$ & 0,114 \\
$\sigma^{2}$ tiempo X constante & 0,016 & - & 0,149 & 0,275 \\
\hline$\sigma^{2}$ Residuo & 0,202 & - & & \\
\hline
\end{tabular}

Fuente: Elaboración propia. En el apartado de efectos fijos, la constante representa el promedio de cortisol en la línea base. En el apartado de efectos aleatorios, la $\sigma^{2}$ constante representa la variabilidad en torno al promedio de cortisol en la línea base. 


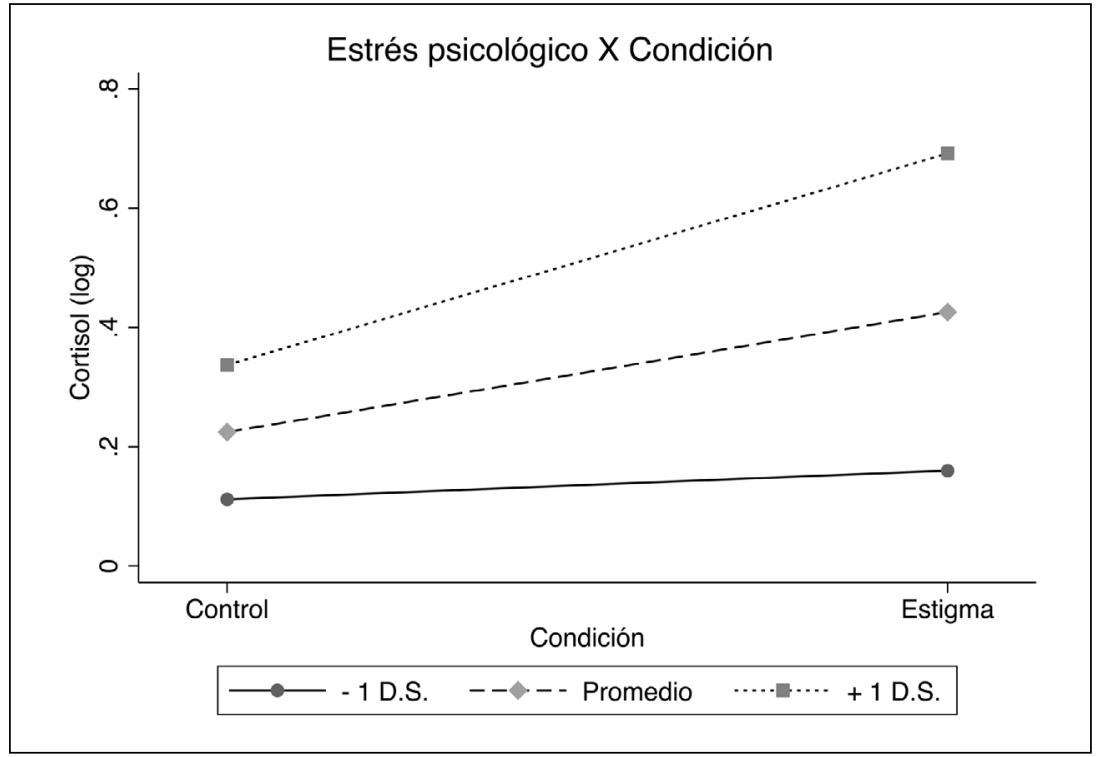

Figura 2. Efecto interactivo de estrés psicológico y condición en el promedio de cortisol. Fuente: Elaboración propia. Las líneas representan el puntaje de estrés psicológico. sis de curvas crecimiento del promedio total de cortisol, el cual varía de modo estadísticamente significativo, tanto al comienzo del estudio como en su cambio en el tiempo. En relación a los predictores de las trayectorias de cortisol, tan solo el estrés psicológico predice una trayectoria positiva de cortisol, tal que por cada punto que éste aumenta, el cortisol se eleva en $0,22 \mathrm{ng} / \mathrm{mL}$ $(p=0,014)$. No existen diferencias estadísticamente significativas en los niveles de cortisol según la condición $(\beta=0,31 ; p=0,097)$. Finalmente, las mujeres con exceso de peso producen menos cortisol que las normopeso $(\beta=-0,41 ; p=0,028)$. $\mathrm{Al}$ incorporar un término de interacción entre la condición y el estrés psicológico, se observa una interacción estadísticamente significativa en el grupo de estigma $(\beta=0,32 ; p=0,005)$, tal que las participantes que puntúan 1 desviación estándar por sobre el promedio en estrés psicológico, producen más cortisol que aquellas que puntúan 1 desviación por bajo el promedio. Estos resultados se mantienen estables controlando por el factor de irritabilidad (Figura 2).

\section{Discusión}

Este estudio tuvo por objetivo determinar una relación causal entre estigma de obesidad, reactividad fisiológica al estrés psicológico, respuesta emocional negativa y la conducta de ingesta alimentaria en una muestra de mujeres adultas. Los resultados demuestran que el estrés percibido se asoció con una mayor ingesta alimentaria, sólo en aquellas mujeres pertenecientes al grupo estigmatizado, resultado concordante con estudios similare $\mathrm{s}^{34,35}$. Este hallazgo es relevante, pues demuestra que el estrés producido por la exposición a situaciones de estigmatización genera mayor ingesta alimentaria, comportamiento no deseado para personas con exceso de peso. En relación al afecto negativo, esta variable no medió la relación antes descrita, pues la introducción de esta variable en el modelo, mantuvo inalterable la relación entre estrés y consumo de alimentos en el grupo estigmatizado. Asimismo, estas emociones no se asociaron con la ingesta, con lo cual no es posible validar, en este estudio, la hipótesis del comfort food, tal como ha sido descrito en estudios similares ${ }^{36,37}$. No obstante esto, se demuestra que la percepción de estrés se relacionó con emociones negativas en ambas condiciones, es decir, mujeres que autorreportaron altos niveles de estrés, independiente de haber sido expuestas al estímulo neutro o de estigmatización, experimentaron mayor irritabilidad, tal como reportan otros estudios ${ }^{31,33,38,39}$. Este resultado es relevante, puesto que al menos en mujeres, una 
consecuencia del estrés es el afecto negativo, por lo que se podría hipotetizar que independiente de la fuente, altos niveles de estrés podrían generar emociones negativas. En consecuencia, es imprescindible que las intervenciones clínicas para la reducción de peso consideren el manejo y afrontamiento de situaciones estresantes y afectos negativos asociados.

Respecto a la reactividad fisiológica al estrés, medida a través del cortisol salival, en este estudio, no hubo diferencias estadísticamente significativas en los niveles de cortisol entre la condición experimental y control, sin embargo, se observa una asociación positiva entre estrés psicológico y cortisol en el grupo de mujeres estigmatizadas y no en el grupo control, lo cual permite establecer un rol moderador del estrés en la asociación entre estigma y cortisol. De esta manera, es posible concluir que las experiencias de estigmatización interactúan con la percepción de estrés, generando una mayor reactividad fisiológica al estrés tal como lo plantea el MCOEP ${ }^{18}$.

Este estudio tiene fortalezas y limitaciones, en primer lugar, es pionero en Chile en estudiar el rol que el estigma de obesidad tiene en relación a la conducta alimentaria en Chile, confirmando los hallazgos obtenidos en estudios foráneos. La metodología y el análisis estadístico empleado, permitió probar relaciones directas e indirectas entre las variables de interés, y evaluar el rol que variables psicológicas tienen en la conducta alimentaria y respuesta fisiológica al estrés. Dentro de sus limitaciones, se debe mencionar que la utilización de videos genera una experiencia de estigmatización indirecta y posiblemente con menor validez ecológica que una situación real de estigmatización, lo cual podría de alguna manera haber influido en la nula asociación entre la condición y la reactividad fisiológica al estrés. Asimismo, otra limitación de este estudio, fue la inclusión de mujeres con peso normal. Si bien, esto fue decidido con la finalidad de generar evidencia local respecto a los efectos del estigma en población general, las investigaciones foráneas en estigma de obesidad se realizan solo con población con exceso de peso, pues se estima que son el principal blanco de estigmatización.

Como futuras líneas de investigación, es importante considerar estudios de diseño experimental que prueben el vínculo entre estigma de obesidad y conducta alimentaria con una ex- periencia de estigmatización directa o en grupos de mujeres solo con exceso de peso u obesidad. Otra variable de interés a incluir en estudios de similares características, es la preferencia alimentaria, ya que en este estudio se consideraron sólo alimentos no saludables. Además, y dado que en este estudio las mujeres de peso normal tuvieron mayores niveles de cortisol que el grupo de mujeres con exceso de peso, sería interesante que futuros estudios comparen la reactividad fisiológica al estrés según IMC.

En síntesis, este estudio demuestra que el estrés psicológico se asocia con ingesta alimentaria en mujeres estigmatizadas. Asimismo, el estrés psicológico modera la relación entre la estigmatización por peso y el promedio de cortisol secretado en esta muestra. En consecuencia, el estigma de obesidad tiene un rol relevante en los procesos fisiológicos, psicológicos y conductuales asociados a obesidad.

\section{Referencias}

1. Imes C, Burke L. The Obesity Epidemic: The USA as a Cautionary Tale for the Rest of the World. Curr Epidemiol Reports. 2014. doi: 10.1007/s40471-014-0012-6.

2. Hruby A, Manson JE, Qi L, Malik VS, Rimm EB, Sun $\mathrm{Q}$, et al. Determinants and consequences of obesity. Am J Public Health 2016; 106 (9): 1656-62. doi: 10.2105/ AJPH.2016.303326.

3. O'Brien PD, Hinder LM, Callaghan BC, Feldman EL. Neurological consequences of obesity. Lancet Neurol 2017; 16 (6): 465-77. doi: 10.1016/S14744422(17)30084-4.

4. Ministerio de Salud. ENCUESTA NACIONAL DE SALUD 2016-2017 Primeros Resultados.; 2017. http:// epi.minsal.cl/wp-content/uploads/2017/11/ENS_201617_PRIMEROS-RESULTADOS.pdf.

5. ONU, OPS. Panorama de La Seguridad Alimentaria y Nutricional; 2017.

6. Moreno M. Definición y Clasificación de la Obesidad. Rev Médica Clínica Las Condes 2012; 23 (2): 124-8. doi: 10.1016/S0716-8640(12)70288-2.

7. Ward-Smith P, Peterson JA. Development of an instrument to assess nurse practitioner attitudes and beliefs about obesity. J Am Assoc Nurse Pract 2016; 28 (3): 125-9. doi: 10.1002/2327-6924.12281.

8. Wright SM, Aronne LJ. Causes of obesity. Abdom Imaging 2012; 37 (5): 730-2. doi: 10.1007/s00261-0129862-x. 
9. Collins J, Bentz J. Behavioral and psychological factors in obesity. J Lancaster Gen Hosp 2009; 4 (4): 124-7.

10. Braghetto I, Taladriz C, Lanzarino E, Romero C. Efecto de la gastrectomía tubular vertical en los niveles de ghrelina plasmática en pacientes obesos 2015; 143: 864-9.

11. Azar A, Franetovic G, Santos H. Determinantes individuales, sociales y ambientales del sobrepeso y la obesidad adolescente en Chile. Rev Med Chile 2015; 143: 598-605.

12. Brewis AA. Stigma and the perpetuation of obesity. Soc Sci Med 2014; 118 (C): 152-8. doi: 10.1016/j.socscimed.2014.08.003.

13. Fruh SM, Nadglowski J, Hall HR, Davis SL, Crook ED, Zlomke K. Obesity Stigma and Bias. J Nurse Pract 2016; 12 (7): 425-32. doi: 10.1016/j.nurpra.2016.05.013.

14. Jackson SE. Obesity, Weight Stigma and Discrimination. 2016; 2 (1): 1-3. doi: 10.4172/2471-8203.100006.

15. Puhl R, Suh Y. Stigma and Eating and Weight Disorders. Curr Psychiatry Rep 2015; 17 (3): 1-10. doi: 10.1007/ s11920-015-0552-6.

16. Friedman K, Reichmann S, Costanzo P, Zelli A, Ashmore J, Musante G. Weight stigmatization and ideological beliefs: Relation to psychological functioning in obese adults. Obes Res 2005; 13 (5): 907-916. doi: 10.1038/ oby.2005.105.

17. Phelan SM, Burgess DJ, Puhl R, Dyrbye LN, Dovidio JF, Yeazel M, et al. The Adverse Effect of Weight Stigma on the Well-Being of Medical Students with Overweight or Obesity: Findings from a National Survey. J Gen Intern Med 2015; 30 (9): 1251-8. doi: 10.1007/s11606-0153266-x.

18. Tomiyama A. Weight stigma is stressful. A review of evidence for the cyclic Obesity/weight-based stigma model. Appetite 2014; 82: 8-15. doi: 10.1016/j. appet.2014.06.108.

19. Latner J, Stunkard A, Wilson G. Stigmatized students: age, sex, and ethnicity effects in the stigmatization of obesity. Obes Res 2005; 13 (7): 1226-31. doi: 10.1038/ oby.2005.145.

20. Boswell R, White M. Gender differences in weight bias internalization and eating pathology in overweight individuals. Adv Eat Disord 2015; 3 (3): 259-68. doi: 10.1080/21662630.2015.1047881.

21. Chen EY, Brown M. Obesity stigma in sexual relationships. Obes Res 2005; 13 (8): 1393-17. doi: 10.1038/ oby.2005.168.

22. Andreyeva T, Puhl RM, Brownell KD. Changes in perceived weight discrimination among Americans, 19951996 through 2004-2006. Obesity 2008; 16 (5): 1129-34. doi: 10.1038/oby.2008.35.

23. Myers A, Rosen JC. Obesity stigmatization and coping: relation to mental health symptoms, body image, and self-esteem. Int J Obes Relat Metab Disord 1999; 23: 221-30. doi: 10.1038/sj.ijo.0800765.

24. Schvey N, Puhl R, Brownell K. The impact of weight stigma on caloric consumption. Obesity. 2011; 19 (10): 1957-62. doi: 10.1038/oby.2011.204.

25. Miller GE, Chen E, Zhou ES. If it goes up, must it come down? Chronic stress and the hypothalamic-pituitary-adrenocortical axis in humans. Psychol Bull. 2007; 133 (1): 25-45. doi: 10.1037/0033-2909.133.1.25.

26. Tomiyama J, Epel E, Poelke G, Kemeny M, Mccoy S, Daubenmier J. Supplemental Material for Associations of Weight Stigma With Cortisol and Oxidative Stress Independent of Adiposity. Heal Psychol 2014; 33 (8): 862-7. doi: 10.1037/hea0000107.supp.

27. Dickerson S, Kemeny M. Acute stressors and cortisol responses: A theoretical integration and synthesis of laboratory research. Psychol Bull 2004; 130 (3): 355-91. doi: 10.1037/0033-2909.130.3.355.

28. Dickerson S, Gruenewald T, Kemeny M. When the social self is threatened: Shame, physiology, and health. J Pers 2004; 72 (6): 1191-216. doi: 10.1111/j.14676494.2004.00295.x.

29. Finch LE, Tomiyama AJ. Stress-Induced Eating Dampens Physiological and Behavioral Stress Responses. Nutr Prev Treat Abdom Obes 2014: 189-195. doi: 10.1016/B978-0-12-407869-7.00018-0.

30. Adam TC, Epel ES. Stress, eating and the reward system. Physiol Behav 2007; 91 (4): 449-58. doi: 10.1016/j.physbeh.2007.04.011.

31. Tomiyama A, Finch L, Cummings J. Did That Brownie Do Its Job? Stress, Eating, and the Biobehavioral Effects of Comfort Food. Emerg Trends Soc Behav Sci 2015; 1-15. doi: 10.1002/9781118900772.etrds0324.

32. Dufey M, Fernández A. Validez y confiabilidad del Positive Affect and Negative Affect Schedule (PANAS) en estudiantes universitarios chilenos. Rev Iberoam Diagnóstico y Evaluación Psicológica 2012; 1 (34): 157-73.

33. Tomiyama A, Dallman M, Epel E. Comfort food is comforting to those most stressed: Evidence of the chronic stress response network in high stress women. Psychoneuroendocrinology 2011; 36 (10): 1513-9. doi: 10.1016/j.psyneuen.2011.04.005.

34. Kwan M, Gordon K. The effects of social support and stress perception on bulimic behaviors and unhealthy food consumption. Eat Behav 2016; 22: 34-9. doi: 10.1016/j.eatbeh.2016.03.024.

35. Tomiyama AJ. Stress and Obesity. Annu Rev 2018; (June 2018): 1-16.

36. Weltens N, Zhao D, Van Oudenhove L. Where is the 
comfort in comfort foods? Mechanisms linking fat signaling, reward, and emotion. Neurogastroenterol Motil 2014; 26 (3): 303-15. doi: 10.1111/nmo.12309.

37. Finch LE, Tomiyama AJ. Comfort eating, psychological stress, and depressive symptoms in young adult women. Appetite 2015; 95: 239-44. doi: 10.1016/j. appet.2015.07.017.

38. Tryon MS, DeCant R, Laugero KD. Having your cake and eating it too: A habit of comfort food may link chronic social stress exposure and acute stress-induced cortisol hyporesponsiveness. Physiol Behav. 2013;114115:32-37. doi: 10.1016/j.physbeh.2013.02.018.

39. Araiza AM, Lobel M. Stress and eating: Definitions, findings, explanations, and implications. Soc Personal Psychol Compass. 2018; 12 (4): 1-13. doi: 10.1111/ spc3.12378. 